Revista de Filología Alemana

ISSN: 1133-0406

https://dx.doi.org/10.5209/rfal.64351

\title{
Lachen nach dem Holocaust: Humor und Versöhnung in Michel Bergmanns Teilacher-Trilogie
}

\author{
Juan Manuel Martín Martín
}

Recibido: 27 de julio de 2018/ Aceptado: 11 de diciembre de 2018

Zusammenfassung. Michel Bergmann vermittelt in seiner Trilogie einen generationenübergreifenden retrospektiven Blick auf die deutsche Nachkriegszeit und auf den Alltag der in Deutschland lebenden Juden. Die Romane zeigen die herausragende Rolle des Humors beim Erinnern an die traumatische Vergangenheit und ebenso für die Lösung der Schwierigkeiten, auf die die Überlebenden in ihrer als fremd wahrgenommenen Umgebung stießen. Aus dem Buch geht hervor, dass der Humor zu einem unerlässlichen Mittel zur Traumabewältigung wird, sodass er eine soziale (Wieder-)Eingliederung der Hauptfiguren in Deutschland begünstigt, zu der auch die ab den 50er Jahren spürbare wirtschaftliche Verbesserung beiträgt. Ihr Leben in der Bundesrepublik bringt eine beginnende Versöhnung mit der Gesellschaft der ehemaligen Feinde mit sich. Sie wird im Wesentlichen auf zweierlei Art gefördert: Einerseits durch die Entstehung von neuen Liebesbeziehungen zwischen Holocaustüberlebenden und (nichtjüdischen) Deutschen; andererseits infolge der von einigen jüdischen Figuren empfundenen Dankbarkeit gegenüber denjenigen, die während des Holocaust ihr Überleben im Untergrund ermöglicht hatten.

Schlüsselwörter: Holocaust; Humor; Traumabewältigung; deutsch-jüdische Literatur; Generationengedächtnis; Michel Bergmann.

\section{[en] To Laugh after the Holocaust: Humor and Reconciliation in Michel Bergmanns Teilacher-Trilogy}

\begin{abstract}
In his trilogy Michel Bergmann shows an intergenerational retrospective look at German postwar era and how Jews established in Germany faced their day by day. These novels reveal how important humor was, not only when dealing with a traumatic past, but also when undertaking the daily difficulties found in an environment they perceived as alien. Humor becomes, as shown in this trilogy, an indispensable resource to face trauma, and in such manner, facilitates the (re)integration of the protagonists in Germany, further helped by the obvious economic improvements beginning in the nineteen fifties. Settling in the Federal Republic of Germany will also bring about an incipient reconciliation with a society that had previously been their enemy. Such reconciliation will be mainly fostered by two key elements: the forging of affective relationships with non-Jewish Germans, and the deep gratitude some of the books' characters feel towards those who helped them survive in secrecy during the Holocaust.
\end{abstract}

Keywords: Holocaust; Humor; Overcoming the Trauma; German-Jewish Literature; Generational Memory; Michel Bergmann.

\footnotetext{
1 Universidad de Salamanca (España)

E-Mail: jm.mm@usal.es
} 


\section{[es] Reír después del Holocausto: humor y reconciliación en la trilogía Teilacher de Michel Bergmann}

Resumen. Michel Bergmann muestra en su trilogía una mirada intergeneracional retrospectiva a la posguerra alemana y al modo en que los judíos establecidos en Alemania afrontaban su día a día. Las novelas revelan el destacado rol que el humor desempeñaba tanto en su manera de recordar el pasado traumático, como en la de resolver las dificultades que a diario se encontraban en un entorno que percibían como ajeno. El humor se convierte, como muestra la trilogía, en un medio indispensable para afrontar el trauma, de tal modo que posibilita la (re)integración de los protagonistas en Alemania, favorecida también por la mejora patente del contexto económico a partir de los años cincuenta. Su establecimiento en la República Federal trae consigo también una incipiente reconciliación con la sociedad que antes habían conformado sus enemigos. Esta será propiciada fundamentalmente por dos elementos: por un lado, la creación de nuevas relaciones afectivas con alemanes no judíos y, por otro, el profundo agradecimiento que sienten algunos de los personajes judíos de la novela por quienes los habían ayudado a sobrevivir en la clandestinidad durante el Holocausto.

Palabras clave: Holocausto; humor; superación del trauma; literatura judeo-alemana; memoria generacional; Michel Bergmann.

Inhaltsverzeichnis. 1. Vergangenheit, Generationen und Familienromane. 2. Betrug als Mittel zum Vorwärtskommen im Nachkriegsdeutschland. 3. Witze als Lebenskampf. 4. Das letzte Paradox: Versöhnung damals und heute. 5. Fazit.

Cómo citar: Martín Martín, J.M., «Lachen nach dem Holocaust: Humor und Versöhnung in Michel Bergmanns Teilacher-Trilogie», Revista de Filología Alemana 27 (2019), 63-80.

\section{Vergangenheit, Generationen und Familienromane}

Michel Bergmann, der Autor der Teilacher-Trilogie, wird 1945 geboren, während seine Eltern in einem schweizerischen Internierungslager für jüdische Überlebende des Holocaust eingesperrt sind. Diese biographische Angabe bestimmt die Generationszugehörigkeit Bergmanns ebenso wie den familiären bzw. kulturellen Kontext, in dem sich sein Leben entfaltet. ${ }^{2}$ Hartmut Steinecke weist auf die „doppelte Realität“ hin, in der die Kinder der Holocaustüberlebenden aufwuchsen, ,in der Vergangenheit der Eltern und in der eigenen Gegenwart" (Steinecke 2005: 8). In diesem Fall befasst sich der Schriftsteller nicht primär mit dem Holocaust selbst sondern mit seinen Nachwirkungen im Nachkriegsdeutschland und mit dem Einfluss der Katastrophe sowohl auf die Überlebenden als auch auf deren Nachkommen. In der Trilogie gibt es allerdings immer wieder Verweise auf den Holocaust, auf den Antisemitismus der Nazizeit oder auf das Exil, die das gegenwärtige Leben der Figuren mit der jüngsten Vergangenheit verbinden. Diese Episoden werden entweder von den Figuren erwähnt oder tauchen einfach als Rückblenden im Laufe der Erzählung auf. Nach Julia Herzberger gibt es einige Themen, die die Texte der zweiten jüdischen Generation in Deutschland bestimmen. Sie erwähnt das deutsch-jüdische Verhältnis in der Gesellschaft, das individuelle Doppelbewusstsein der nachgeborenen Generation und, "damit eng verbunden [,] auch die Frage nach der eigenen Identität: jüdisch,

2 Vgl. Herzberger (2009: 1): „Die nachgeborenen Generationen suchen nach einer Vergangenheit, die ihnen oft unbekannt ist, dennoch weitreichende Folgen für ihre eigene Biografie und Persönlichkeitsfindung mit sich bringt." 
deutsch oder beide zu sein" (Herzberger 2009: 17-8). Das alles findet sich in der Teilacher-Trilogie, ${ }^{3}$ aber darüber hinaus ist die intensive Verwendung des Humors ein relevantes Unterscheidungsmerkmal gegenüber anderen Werken. Der Standpunkt Bergmanns ist nur in einem konkreten historischen und kulturellen Kontext verständlich, in dem der Holocaust mit neuen vielfältigen Bedeutungen ergänzt worden ist. Hinzu kommt, dass die Generation der Eltern und die von ihnen verursachten Hemmungen nicht mehr existieren. Unabhängig davon ist das Thema des Völkermords, insbesondere im deutschsprachigen Raum, immer noch konfliktgeladen, vor allem wenn es aus Perspektiven behandelt wird, die von dem allgemein Akzeptierten abweichen. Um einen ausgewogenen Diskurs zu vermitteln, wird in der Trilogie auf Verweise und Anspielungen zurückgegriffen, die den Holocaust in den Mittelpunkt rücken.

Ein Ziel dieses Artikels ist die Kontextualisierung der Trilogie in einen historischen bzw. kulturellen Rahmen. Neben einer literaturwissenschaftlichen Analyse geht es hier darum, die mit der Thematik und der Gattungszugehörigkeit des Werkes verknüpften unterschiedlichen Aspekte miteinander in Verbindung zu bringen. Auf diese Weise kann die volle Bedeutung der Trilogie Michel Bergmanns erfasst werden: Wer hätte sich denn noch getraut, einen Roman über Juden zu schreiben, die Nichtjuden betrügen, oder die Witze über Juden erzählen, die alle möglichen Stereotypen enthalten? Das Trauma der Überlebenden bleibt meistens im Hintergrund, denn die Figuren wollen einfach in die Zukunft blicken und ihr neues Leben in den Griff bekommen: „Juden und Deutsche - sie wollten nur eines: nach vorne schauen, das Leben genießen - wenngleich auch aus völlig unterschiedlichen Gründen. Das deutsche Wirtschaftswunder kam den Juden in diesem Lande nicht ungelegen“ (Schneider 2000: 19-20). Letzten Endes dürfen sie nach 1945 wieder eine relativ normale Existenz haben. ${ }^{4}$ Im Hinblick auf die wirtschaftliche Erholung, die das Wirtschaftswunder Jahre später darstellen würde, hatte der Kanzler Konrad Adenauer schon in seiner ersten Regierungserklärung eine klare Absicht verkündet: „Vergangenes vergangen sein lassen“ (Adenauer 1949). Dieses politische Statement ließ die offizielle Position gegenüber der jüngsten Vergangenheit durchblicken. Aber wie die Trilogie aufzeigt, konnte Adenauers Wunsch keinesfalls die Erinnerungen verblassen lassen, die der deutschen Bevölkerung, und insbesondere den jüdischen Überlebenden im Gedächtnis geblieben waren. Michel Bergmann verfügt über eine geeignete Position, die ihm ermöglicht, diese Fiktion mit autobiographischem Hintergrund zu verwirklichen. Dieser deutschsprachige jüdische Schriftsteller kennt sich gut in diesem Bereich aus, und das trägt dazu bei, die Authentizität und Glaubwürdigkeit des Romans zu sichern. Gerade in Deutschland, wo sich neue bzw. unübliche Blicke auf die jüngste Geschichte oft auf einer Gratwanderung befinden.

Neben diesen Aspekten ist beachtenswert, dass die Teilacher-Trilogie größtenteils ins Genre der Familien- oder Generationenromane eingeordnet werden kann.

3 Eine der Figuren der Trilogie bedauert explizit, dass die erste Generation der in Deutschland gebliebenen Überlebenden ihre Kinder in Schwierigkeiten gebracht hat, denn ,[sie] haben ihnen keine Chance gegeben, hier Wurzeln zu schlagen. Sie sind mit dem gepackten Koffer groß geworden“ (Bergmann 2011: 205).

4 Bei der Suche nach der Normalität hat das Lachen eine wesentliche Funktion, denn es trägt dazu bei, die vergangenen Erfahrungen in gewisser Hinsicht zu mildern. Die Überlebenden greifen in der Trilogie nicht zuletzt auf den Humor zurück, um den Anschein einer gewöhnlichen Existenz zu erreichen. Das bedarf allerdings der vorläufigen Unterdrückung der Empfindungen zugunsten einer pragmatischen Einstellung gegenüber ihrem gegenwärtigen Leben. 
Diese Gattung erlebt nach 1990 einen Boom, nicht nur unter den jüngsten Autoren (der Generation der Enkelkinder) sondern auch unter denjenigen, die ihnen vorangehen. Diese hatten die Väterliteratur der 70er und der 80er Jahre als Aufarbeitung der traumatischen jüngsten Geschichte entwickelt; ihre Werke brachten damals also einen kritischen Blick auf die Vergangenheit mit sich. Auch der Umgang der jüdischen Autoren der zweiten Generation mit dem Holocaust war problematisch und oft gekennzeichnet durch Vorwürfe gegen die Eltern und deren Beziehung zur Vergangenheit. ${ }^{5}$ Die aktuellen Generationenromane beinhalten genauso wie früher die Väterliteratur Bezüge auf vergangene Ereignisse, es gibt jedoch einen wesentlichen Unterschied, wie Aleida Assmann betont:

Während die Väterliteratur im Zeichen des Bruchs steht - ihr thematisches Zentrum ist die Konfrontation, die Auseinandersetzung, die Abrechnung mit dem Vater, steht der Familienroman im Zeichen der Kontinuität - hier geht es um die Integration des eigenen Ich in einen Familienzusammenhang, der andere Familienmitglieder und Generationen mit einschließt. Im Familienroman gewinnt die Identitätssuche eine historische Tiefe und Komplexität, die in der Väterliteratur noch nicht angelegt ist (Assmann 2006a: 26). ${ }^{6}$

Wie oben dargelegt, gab es literarische Werke, die im Zusammenhang mit den unterschiedlichen und oft widersprüchlichen Erfahrungen und Sorgen der Nachkommen - der Täter oder auch der Opfer - entstanden. Sie entsprachen der kritischen Vision der Kinder der Zeitzeugen in Bezug auf die Erlebnisse ihrer Eltern und vor allem auf deren Umgang mit jenen Vorkommnissen. Im Gegensatz dazu vertritt der Familienroman die Interessen der einen und der anderen Seite, denn alle Autoren suchen nach einem persönlichen Platz in der familiären Geschichte, was gleichzeitig in hohem Maße zur Gestaltung der eigenen Identität beiträgt. Die Autoren der Generationsromane sind sehr verschieden mit in der Regel völlig unterschiedlichen historischen Referenzen: Diesbezüglich könnte man zwischen deutschsprachigen jüdischen und nichtjüdischen Autoren unterscheiden. ${ }^{7}$ Michel Bergmann vermittelt aus der Perspektive der zweiten jüdischen Generation einen versöhnlichen Blick auf die traumatische Vergangenheit, ebenso wie die österreichische Autorin Eva Menasse ${ }^{8}$ aus der

5 In diesem Sinne entsteht unter den jüdischen Autoren gewissermaßen ein paralleler Vorgang zu dem, was generell in der Gesellschaft geschieht. Die Vorwürfe der jüdischen Literatur erfolgen, während die Väterliteratur die Reaktion der nichtjüdischen Schriftsteller auf die tadelnswerte Einstellung ihrer Eltern schildert, die die Figur des Täters oder des Mitläufers verkörpern (Steinecke 2005: 12).

6 Dieser Aspekt soll mit dem Standpunktwechsel nachfolgender Generationen zum Thema Holocaust in Verbindung gebracht werden. Unter den überlebenden Opfern entwickelte sich allmählich ein Erinnerungswille, aber demgegenüber war unter den Tätern der ersten Generation das Schweigen die Regel (auf diese Einstellung reagieren deren Nachkommen beispielsweise durch die Väterliteratur). In Bezug auf diese für (nichtjüdische) deutsche Zeitzeugen typische Sprachlosigkeit hebt Assmann hervor: „Es gibt allerdings Anzeichen dafür, dass sich das nach dem Ableben der Tätergeneration in der späten zweiten und dritten Generation ändert“ (Assmann 2006b: 214).

7 Unter den nichtjüdischen deutschsprachigen Autoren können auch ein paar Beispiele erwähnt werden: Sowohl Dieter Forte (zweite Generation) als auch Tanja Dückers (dritte Generation) suchen in ihren jeweiligen Romanen Tetralogie der Erinnerung bzw. Himmelskörper nach einer Versöhnung mit der familiären Vergangenheit, die ihre eigene Identität festigt oder definiert.

8 In Vienna (2005) vermittelt die Autorin ein generationenübergreifendes Porträt einer Wiener Familie, in der jüdische und nichtjüdische Wurzeln zusammenfließen. Das Thema Judentum ist von großer Bedeutung im Roman, allerdings nicht nur für die Darstellung der Nazi-Zeit, sondern auch für die Gestaltung der Betrachtungsweise der Enkelgeneration, aus deren Perspektive erzählt wird (Jahn 2006: 588-589). 
Perspektive der dritten jüdischen Generation. Beide „suchen nach einer Vergangenheit, die ihnen oft unbekannt ist, dennoch weitreichende Folgen für ihre eigene Biografie und Persönlichkeitsfindung mit sich bringt" (Herzberger 2009: 1).

\section{Betrug als Mittel zum Vorwärtskommen im Nachkriegsdeutschland}

Die Trilogie besteht aus den Romanen Die Teilacher, Machloikes und Herr Klee und Herr Feld. ${ }^{9}$ Die Rückblenden spielen jeweils eine zentrale Rolle, denn sie ermöglichen, Geschehnisse unterschiedlicher Zeiten zu kombinieren, und betonen hiermit, wie die Vergangenheit das Leben der Figuren nachträglich bestimmt. Für den Titel der zwei ersten Teile der Trilogie hat Michel Bergmann jiddische Wörter gewählt, ${ }^{10}$ was schon darauf hinweist, welches Gewicht diese Sprache im Roman hat. Der erste Band heißt Die Teilacher (jiddisches Wort für Vertreter des Einzelhandels) und der zweite Machloikes (jidd. für Machenschaften, Ärger). Das Jiddisch verkörpert die Spur einer verloren gegangenen Welt, der die Hauptfiguren angehörten, und die ihre Identität prägte, bevor alles zerstört wurde. Der Gebrauch von jiddischen Ausdrücken ermöglicht die Entstehung eines Zusammengehörigkeitsgefühls unter den Teilachern, die versuchen, sich im Nachkriegsdeutschland durchzuschlagen. Außerdem vermittelt das Jiddische oft witzige Inhalte in Form von Songs, Witzen oder Wendungen, mit deren Hilfe die Überlebenden der schwierigen Lage in der Nachkriegszeit entgegengetreten.

Anlässlich des Todes David Bermanns, eines herausragenden Teilachers, versammeln sich 1972 im ersten Band der Trilogie einige seiner ehemaligen Kollegen und Alfred Kleefeld, dessen Nennonkel der Verstorbene war. Bei dieser Gelegenheit denken sie an ihre Erlebnisse im Nachkriegsdeutschland zurück. Damals trafen sich alle in Frankfurt am Main, nachdem sie aus dem Exil oder aus unterschiedlichen Konzentrationslagern zurückgekommen waren. Es ging ihnen immer noch ums Überleben, denn um sich herum fanden sie ein ruiniertes Land, wo sie mit einem strapaziösen Alltag kämpfen mussten. Allerdings war das nur eine provisorische Situation, denn die meisten dachten an Auswanderung, an das Verlassen des Landes, das letztendlich hinter ihrem Unglück steckte: „So wie die meisten Juden im Deutschland von damals hatte er [Max Holzmann] vor, ein paar Jahre zu bleiben, etwas Geld zu machen und später irgendwohin auszuwandern" (Teilch: 110). ${ }^{11}$ In den ersten Nachkriegsjahren lebten in Deutschland etwa eine Viertel Million jüdische Überlebende, deren Herkunftsländer überwiegend in Osteuropa lagen (Brenner 1995: 10). Allerdings hatten sie überhaupt keine Absicht, in die Heimat zurückzukehren, sondern sie wollten im westlichen Teil des geteilten Deutschlands unter dem Schutz der Alliierten bleiben - die meisten von ihnen jedoch nur vorübergehend, denn sie planten in die USA oder nach Israel zu emigrieren. Diese erhebliche Präsenz von Juden im

9 Eine Verfilmung aus dem Jahr 2017 basiert auf den ersten zwei Teilen der Trilogie, ihr Titel ist Es war einmal in Deutschland. Im Film werden die zentralen Aspekte der beiden Romane aufgenommen: Einerseits die abenteuerliche Tätigkeit der Teilacher, und andererseits die unglaubliche Aufgabe, die eine Hauptfigur der Geschichte erfüllen muss.

10 Jeder Band der Trilogie enthält ein Glossar mit den jiddischen Wörtern, die im Roman verwendet werden.

11 Die Romane werden künftig folgendermaßen zitiert: Teilch (Die Teilacher), Machl (Machloikes) und KuF (Herr Klee und Herr Feld). 
ehemaligen Land der Täter könnte paradox erscheinen, ${ }^{12}$ aber genau dieser Ort wurde unter den damaligen Verhältnissen zum sichersten Zufluchtsort in Europa. ${ }^{13}$ Die Teilacher aus dem Roman heißen Max Holzmann, Emil Verständig, Jankel Lubliner, Robert Fränkel, Jossel Fajnbrot, Samuel Honigbaum, Willy Serafin oder Moishe Krautberg und kommen aus allen Ecken des Kontinents, wo ihre Leute beinahe ausgerottet worden waren. Wie bei den traditionellen Schelmen die Regel ist, sind die meisten von diesen Figuren allein auf der Welt, und zwar aufgrund des Völkermords. Um ihren Lebensunterhalt zu verdienen, greifen sie auf einen Beruf zurück, dem vor dem Krieg oft Juden nachgingen. Das war aber nicht gerade ihre Traumtätigkeit:

Die Teilacher der Nachkriegszeit waren Gestrandete. Sie alle hatten die Idee gehabt, Warenhausbesitzer, Wundergeiger, Architekt, Anwalt oder Arzt zu werden, aber der Führer wusste dies zu verhindern. So sahen sie ihren Beruf als eine Art von vorübergehendem, schicksalhaftem Ereignis auf dem steinigen Weg zu etwas ganz anderem, etwas Besserem (Teilch: 104).

Die Hauptfiguren des Romans sind also zunächst keine Sieger, sie entsprechen in vielerlei Hinsicht dem Bild eines Schelms, einer Figur am Rand der Gesellschaft; sie „wurden als niedere Kaste angesehen“ (Teilch: 104). Wie das Zitat oben zeigt, sind die Teilacher aufgrund der außergewöhnlichen Umstände meistens ungebildet und greifen auf einen Beruf zurück, durch den sie ihre Probleme in den Griff bekommen können. ${ }^{14}$ Sie benötigen Waren für den Verkauf und Kunden, denen sie die Produkte anbieten können; diese Rolle wird von den Frankfurtern übernommen, die unter mühsamen Bedingungen leben müssen und ihre eigenen Schwierigkeiten und Gewissensbisse überwinden wollen. Moralische Vorbehalte gegen Betrügerei kommen nicht in Frage, denn die heutigen Opfer waren bis 1945 die Täter, sodass der Betrug $\mathrm{zu}$ einer Art Rache wird. Im Roman wird auch auf die fragwürdige Beziehung der Deutschen zu den Juden vor der Nazi-Zeit hingewiesen. Nachdem der Krieg und die Verfolgung schon zu Ende waren, erinnert sich eine der Hauptfiguren an Warnungen, die eine tatsächliche Integration der Juden bezweifelten. So werden die Worte des deutsch-jüdischen Autors Jakob Wassermann (1873-1934) wiedergegeben, die er im Rahmen einer Podiumsdiskussion ausgesprochen hatte. Wassermann ist der Autor von Mein Weg als Deutscher und Jude (1921), einem autobiographischen Essay, in dem er seine diffizile deutsch-jüdische Doppelexistenz skizziert. ${ }^{15}$ Der Bezug auf

12 In ihrem Buch über die Beziehungen zwischen Juden, Deutschen und Alliierten im Nachkriegsdeutschland stellt Atina Grossmann heraus, dass viele Autoren schon auf diese Ironie der Geschichte hingewiesen haben (Grossmann 2007: 134).

13 Sogar in Ortschaften, „die die Nazis nicht ,judenrein’ machen mußten, da dort niemals Juden gelebt hatten, entstanden nun jüdische Schulen und Sportklubs“ (Brenner 1995: 10).

14 So wird eine der Figuren des Romans beschrieben: „Willy war ein notorischer Lügner, der einzige Mensch auf der Welt, der sich sechsmal in einem Satz widersprechen konnte, ohne das Gegenteil zu behaupten. Aber jede Unwahrheit verkaufte er mit so viel erdrückendem Charme und gnadenloser Unwiderstehlichkeit, dass es seinen Opfern, vor allem seinen zahlreichen Gläubigern, schwerfiel, ihn zu hassen“ (Teilch: 71).

15 Auf die in diesem Werk dargestellten Ideen greift der Autor der Trilogie deutlich zurück, um den Standpunkt Wassermanns zu schildern und ebenso um die damalige Situation der Juden in Deutschland zu charakterisieren: „Das »Entreeticket« zum Deutschsein vergeben die Deutschen selbst, und sie entscheiden, wer es bekommt, und das sind niemals die Juden. Bin ich selbstbewusst, sagen sie, schau an, der arrogante Jud, bin ich bescheiden, sagen sie, sieh nur, der Jud, wie er sich einschmeichelt, bin ich reich, sagen sie, der jüdische Betrüger, bin ich arm, sagen sie, der Schmarotzer - ich kann machen, was ich will, ich bin und bleibe für sie nur der Jud!“ (Teilch: 48-9). 
reale Persönlichkeiten in der Teilacher-Trilogie verleiht der Fiktion einen historischen Rahmen, der dazu beiträgt, die Handlung kohärenter zu machen. Als die Hauptfigur David Bermann in Paris lebt, lernt er Herschel Grynszpan kennen, der später den deutschen Diplomaten Ernst von Rath ermordet und dadurch die Ereignisse der Reichskristallnacht auslöst (Teilch: 79). Außerdem kennt Bermann den deutschen Schriftsteller Soma Morgenstern, der damals Journalist bei der Frankfurter Zeitung war (Teilch: 44). Darüber hinaus trifft Robert Fränkel, die zentrale Figur des Romans Machloikes, als er von der Gestapo inhaftiert wird, historische Persönlichkeiten wie den Pastor Martin Niemöller, den Hitler-Attentäter Georg Leser und den oben erwähnten Herschel Grynszpan (Machl: 60). Aus dem Kontext kann man außerdem erschließen, dass er Kurt Tucholsky persönlich kannte (Machl: 61).

Oft mussten die Juden in ihre DP-Lager (Displaced Persons Camps) fliehen, wenn die Kunden mit den von ihnen abgeschlossenen Schwarzmarktgeschäften nicht zufrieden waren (Teilch: 111). Der historische Kontext liefert zumindest eine Rechtfertigung für die zum Teil rechtswidrigen Handlungen, die von den Teil-achern begangen werden: „Wer den Tod überlebte, in Auschwitz seine Kindheit verloren hatte, der war verdorben, war mitleidlos, war ein Raubtier, das nur an sein Überleben dachte. Das war die Erfahrung, die einer wie Jankel aus der Hölle mitbrachte. Und Jankel war nicht allein" (Teilch: 177-178). Jahre später werden die DP-Lager geschlossen, aber einige der ehemaligen Insassen bleiben noch immer in Deutschland. Ihr Verbleiben bedeutet auch einen Racheakt an den Tätern:

Die Deutschen haben uns gewollt? Deutschland war Feindesland! Wir waren für sie das jeden Tag sichtbare schlechte Gewissen. Wir Überlebenden erinnerten sie an die Toten. (...) Unsere Leute im Ausland konnten es nicht verstehen, dass wir nicht ausgewandert waren. Meinst du, es hat Spaß gemacht, sich immer rechtfertigen zu müssen? Aber ich [Robert Fränkel] persönlich habe mir dann immer gesagt, ich lebe und bin die jüdische Rache an Hitler (Teilch: 204).

Im westlichen Teil des kurz zuvor geteilten Deutschlands fühlen die Teilacher, woher auch immer sie herkommen, dass sie eine besondere Gemeinschaft bilden. Im Prinzip wollen sie dort nur kurzzeitig bleiben, weil Deutschland eine Durchgangsstation ist, aber auf jeden Fall brauchen sie Geld, um die Reise fortsetzen zu können. Daher müssen die Waren verkauft werden, egal wie. Die Hauptfiguren des Romans sprechen nur von vagen Auswanderungsplänen, denn die meisten haben unbewusst erkannt, dass ihre Zukunft sich in Deutschland befindet. Bereits in den 50er Jahren war Deutschland für viele Juden keine vorläufige Station, selbst wenn die Entscheidung erst Jahre später in Worte gefasst wurde. Das hing mit einem latenten Schuldgefühl zusammen, das man lange verspürte: „Das schlechte Gewissen, im Land der Mörder zu leben und nicht in die eigentliche Heimat des jüdischen Volkes auszuwandern, bestimmte daher auch für Jahrzehnte die Identität der Juden im Nachkriegsdeutschland" (Brenner 1995: 203-4).

In diesem Kontext verwenden die Teilacher allerlei Methoden, um an ihr Ziel zu kommen, unabhängig davon, ob sie dafür die Kunden betrügen müssen. Eigentlich geht es überwiegend um Gaunereien, bei denen sie von der unangenehmen Lage der Bevölkerung profitieren: David Bermann und Willy Serafin studieren die Todesanzeigen der Zeitungen, um die Witwen der Verstorbenen davon zu überzeugen, dass ihre Ehemänner kurz vor dem Tod eine Bestellung aufgegeben hatten. Noch unter 
Schock akzeptieren die Frauen dann diesen letzten Wunsch und bezahlen die angebliche Bestellung. Die Teilacher zeigen also kein Mitleid mit diesen Frauen, die sich in einer traurigen Situation befinden. Schließlich beobachten sie als Juden die Realität von außen, denn die Betrogenen leben - als Deutsche - in einer Gesellschaft, zu der die Betrüger nicht ganz gehören. Als sein Kollege Emil Verständig die Familie eines im Krieg gefallenen Soldaten betrügt, fühlt der Teilacher Moishe Krautberg zunächst rachmunes (jidd. für Mitleid), ,,aber dann fiel ihm ein, dass die Deutschen selbst daran schuld waren, an ihrem Schicksal“" (Teilch: 156). ${ }^{16}$

Oft sind die Teilacher einfach eine Art Marktschreier, die für den Verkauf ihre Schauspielkunst anwenden:

Herrliche Ware, Weißwäsche, Kattun, Damast, Leinen, Spitze, fühlen Sie, fassen Sie es an, na? Ist das ein Frottee? Ah, und wenn Sie jetzt den Preis hören, werden Sie mich für meschugge halten, Sie werden mich abholen lassen ins Irrenhaus. Wenn ich Ihnen sage, was soll ich Ihnen sagen, der Stoff kostet mich allein schon... beim Augenlicht meiner Frau, wissen Sie was, aber Sie sagen es zu keinem nicht weiter, meine Kollegen steinigen mich, also nennen Sie einen Preis, und ich akzeptier!

So redete man mit diesen sperrigen Deutschen, und schließlich kauften sie und kauften, und jedes Aussteuerpaket, das zwischen Winter '45 und Winter '65 in einem deutschen Kleiderschrank oder in Omas Erbtruhe mottensicher verstaut wurde, war durch die Hände eines fleißigen Teilachers gegangen, schwer verkauft mit Lachen, Lügen und Weinen (Teilch: 105).

Es geht also ums Vorankommen, was auch immer die Mittel dafür sind. Die Teilacher versuchen, die Vergangenheit hinter sich zu lassen, die traumatischen Erfahrungen zu vergessen und, oft unbewusst, ein relativ normales Leben im Land der Verbrecher zu führen. ${ }^{17}$ Auch später, als einige Teilacher nicht mehr von Tür zu Tür gehen müssen und ihre eigenen Geschäfte eröffnet haben, endet der Betrug nicht: Robert Fränkel verkauft in seinem Teppichladen Waren aus Jutana, Sisala oder Cottonia, alles Orte, die gar nicht existieren, aber er überzeugt die Kunden davon, diese wertvollen Produkte eifrig kaufen zu wollen (Machl: 69). Fränkels Schelmennatur bleibt also erhalten, auch nachdem die schwierigsten Zeiten schon vorbei sind; nach wie vor wird also sein Recht beibehalten, an dem Volk der Täter eine „milde“ Rache

16 Der damalige Opferdiskurs der Deutschen, der die Nachkriegszeit kennzeichnete, wird auch im Roman geschildert, vor allem mit Verweisen auf die durch den Luftkrieg zerstörten Städte und auf die von den Russen begangenen Massenvergewaltigungen.

17 Es war nicht immer einfach, dieses normale Leben zu führen, denn die Juden wurden oft mit der aufreizenden Haltung der Behörden konfrontiert. Ebenso wie in anderen Romanen jüdischer Autoren, die sich mit der Nachkriegszeit auseinandersetzen, werden hier die Schwierigkeiten geschildert, die ein Antrag auf Wiedergutmachung mit sich bringt: „Der Antragsteller muss belegen, was ihm oder seiner Familie wann gehört hat, und den Verlust beweisen! Ferner, wie viele seiner Verwandten wo, wie und warum ums Leben gekommen sind. Ob es Zeugen oder Dokumente über deren Ansprüche gibt. Und das, nachdem das gesamte jüdische Vermögen arisiert und Hunderttausende enteignet wurden. Und die Milliarden, die Juden vor 1933 in die Versicherungssysteme eingezahlt haben? Wo werden die berücksichtigt? Oder was ist mit den Familien, die komplett ausgerottet wurden? Wo niemand mehr klagen kann?“ (Machl: 148-149). Das gleiche Erlebnis muss die zentrale Figur des Romans Familienleben (Viola Roggenkamp, 2004) durchmachen: Ihr Antrag auf Wiedergutmachung wird von den Justizbehörden einfach abgelehnt, was bei ihr eine Lebenskrise auslöst, denn sie muss ihre Beziehung zur Tätergesellschaft wieder neu bewerten. 
zu nehmen. Dafür benötigt man ohne Zweifel einen großen und vielleicht auch atypischen Sinn für Humor: ,Wir [die Juden] sind so toll, dass wir sogar witzeln, wo wir weinen müssten. Wir belustigen uns an unserer eigenen Angst, um sie mit dem Allheilmittel des Gelächters wegzutherapieren“ (Joffe 2015: 49). Eben deshalb spielen Witze in der Trilogie eine besondere Rolle, vor allem im ersten und im zweiten Band, in denen die Nachkriegszeit geschildert wird.

\section{Witze als Lebenskampf}

Obwohl der Kantor einer Frankfurter Synagoge im Roman behauptet, dass nach dem Holocaust der Humor gestorben und das Lachen für immer verloren seien (Machl: 221), ${ }^{18}$ werden Witze immer mal wieder in die Gespräche der Teilacher miteinbezogen. Es wird sowohl auf jüdische Witze als auch auf Judenwitze zurückgegriffen, ${ }^{19}$ deren Inhalt sich auf die klassischen negativen Vorurteile gegenüber Juden bezieht: „Kennen Sie den? Wissen Sie warum so viele Juden im KZ sind? ...? ... Weil's nix kostet!“ (Teilch: 118). ${ }^{20}$ Noch ein Beispiel:

Du weißt, warum es zehn Gebote sind?

Nein.

Gott sagte: Ich habe Gebote für euch. Und Moses fragte, was kosten die? Nix, sagte Gott. Okay, dann nehme ich zehn! (Teilch: 30).

In die Geschichte werden auch Verweise auf angeblich lächerliche Eigenschaften der Juden aufgenommen: „Krautberg kam näher. Zuerst kam seine lange, krumme Nase in den Raum, dann lange nichts, dann der Rest“ (Teilch: 144). Die sogenannten Nasen-Witze sind für Josef Joffe keine jüdischen Witze, sondern antijüdische Witze, die rassistische Klischees aufnehmen (Joffe 2015: 25). Das Aussehen Krautbergs wird im Roman sogar mit einer „Stürmer-Karikatur eines Juden“ (Teilch: 144) verglichen, was die herkömmlichen Rassenvorurteile bestätigt. Diese antisemitischen Andeutungen werden in der Trilogie jedoch gleichzeitig inaktiviert, wenn die Betroffenen selbst über die Klischees lachen. Außerdem enthalten die Scherze der Teilacher nicht nur negative Stereotypen, sie verweisen auch auf die jüdische Natur: „Die Männer stritten über ein Thema und am Ende behielt jeder recht. Zwei Juden, drei Meinungen, wie David einmal so treffend bemerkte“ (Machl: 9). Es gelingt den Teilachern, ein ernstes Gespräch in eine witzige Konversation zu verwandeln: „Alles ist relativ, wollte Einstein damit sagen. Schau, rauchen eine Zigarette ist a [jidd. für ein] kurzes Vergnügen, relativ. Es dauert zehn Minuten. Aber zehn Minuten sitzen mit dem tuches [jidd. für Arsch] auf einer heißen Herdplatte ist relativ lang!“ (Machl: 24). Die Figur der jüdischen Mutter bzw. Ehefrau ist auch produktiv für die Entstehung von

18 Diese Behauptung soll mit Adornos Aussage über die Unmöglichkeit der Dichtung nach Auschwitz (1951) in Verbindung gebracht werden, denn beide gehen davon aus, dass die Welt nach dem Völkermord in neue Referenzrahmen eingesetzt wird, die neuer Grundlagen und Handlungsformen bedürfen

19 Zur Unterscheidung zwischen jüdischen Witzen und Judenwitzen, siehe Joffe 2015: 24 ff.

20 Dieser Witz wird auch in Machloikes erzählt, in diesem Fall aber direkt von einem SS-Mann im Konzentrationslager Sachsenhausen, wo die Hauptfigur Robert Fränkel inhaftiert ist (Machl: 80). 
Witzen; Beispiele dafür sind auch im Roman zu finden: „Mama, ich habe eine tolle Rolle in unserem neuen Schulstück bekommen. Nu, was spielst du?, fragt die Mutter. Ich spiele einen jüdischen Ehemann. Was?, schreit die Mutter, keine Sprechrolle?!“ (Machl: 207). Darüber hinaus bieten auch religiöse Inhalte Stoff für jüdische Witze: ,Willst du Gott zum Lachen bringen, dann mach einen Plan!“ (Machl: 214). Außerdem wird oft auf den scharfen Verstand hingewiesen, der seit Jahrhunderten den Juden zugeschrieben wird:

Ein alter Jude liegt im Sterben und verlangt nach dem Pfarrer. Die Familie ist entsetzt. Aber der Alte will keinen Rabbiner sehen, sondern einen katholischen Priester, denn er hat vor, auf dem Sterbebett zu konvertieren. Die Familie ist außer sich.

Du warst doch immer ein guter, gläubiger Jude gewesen, sagen sie, warum willst du jetzt übertreten? Da sagt der Alte verschmitzt: Es ist doch besser, ein goj stirbt, als ein jid! (Teilch: 260).

Diese Witze waren eigentlich nichts Neues, sondern eine Fortsetzung von dem, was in den KZs erzählt worden war. Der zur NS-Zeit in Auschwitz inhaftierte Wiener Psychiater Viktor Frankl (1905-1997) bezieht sich in seinem Erinnerungsbuch ...trotzdem Ja zum Leben sagen. Ein Psychologe erlebt das Konzentrationslager (1977) darauf:

Ist es schon erstaunlich genug für den Außenstehenden, daß es im Konzentrationslager so etwas wie Natur- oder Kunsterleben gibt, so mag es noch erstaunlicher klingen, wenn ich sage, daß es dort auch Humor gibt. Freilich: wiederum nur in Ansätzen, und wenn dann natürlich nur für Sekunden oder Minuten. Auch der Humor ist eine Waffe der Seele im Kampf um ihre Selbsterhaltung. Ist es doch bekannt, daß der Humor wie kaum sonst etwas im menschlichen Dasein geeignet ist, Distanz zu schaffen und sich über die Situation zu stellen, wenn auch nur, wie gesagt, für Sekunden (Frankl 1982: 74). ${ }^{21}$

Damals bedeutete der Humor vielleicht ein Mittel zum Überleben und später, nachdem der Krieg zu Ende war, wurde er einfach ein Heilmittel, das dazu beitragen sollte, die eigenen Traumata zu überwinden. ${ }^{22}$ Diesbezüglich behauptet die amerika-

21 Frankl berichtet über verschiedene Situationen, bei denen das Komische im Lager eine wichtige Rolle unter den Gefangenen spielte. Obwohl ihre Ankunft im Lager außergewöhnlich war, reagierten die Häftlinge oft auf eine herkömmliche Art darauf: Zum Beispiel, als sie nach der Selektion witzige Bemerkungen in den Duschen machten: „So zerrann eine Illusion nach der andern, die der eine oder andere von uns noch behalten haben mochte. Jetzt überkommt die meisten von uns aber ein irgendwie Unerwartetes: Galgenhumor! Wir wissen, wir haben nichts mehr zu verlieren außer diesem so lächerlich nackten Leben. Während schon die Brause fließt, rufen wir einander mehr oder weniger witzige, auf jeden Fall witzig sein sollende Bemerkungen zu und bemühen uns krampfhaft, vor allem über uns selbst, dann aber auch über einander uns lustig zu machen. Denn, nochmals: es kommt Wasser aus den Brausetrichtern!...“ (Frank1 1982: 34-35).

22 Während des Holocaust, ebenso wie später in der Nachkriegszeit, hatten diese Witze die soziale Funktion, die Henri Bergson dem Lachen zugeschrieben hatte: „Um das Lachen zu verstehen, müssen wir es wieder in sein angestammtes Element versetzen, und das ist die Gesellschaft; wir müssen seine nützliche Funktion bestimmen, und das ist die soziale Funktion (...) Das Lachen muß gewissen Anforderungen des Gesellschaftslebens genügen. Das Lachen muß eine soziale Bedeutung haben“" (Bergson 2011: 17). 
nische Psychotherapeutin Jacqueline Garrick: „During the trauma, humor may have been a tool of survival; in the recovery process, it can be used as a tool for surviving and thriving as well"“ (Garrick 2006: 181). In Machloikes erzählt Robert Fränkel in Bezug auf seine Inhaftierung in Sachsenhausen: „Ich erfand Witze und probierte sie morgens im Waschraum bei den anderen aus. Alle lachten" (Machl: 82). Der Witz ergibt „eine feine Gesprächstherapie“ (Joffe 2015: 38), die für die Betroffenen ${ }^{23}$ eine heilende Funktion haben kann; in diesem Sinne ist der Humor - als Witz verwirklicht - „die verbale Waffe des Schwächeren, der sehr wohl weiß, dass er nicht die Macht hat, sich direkt zu wehren; also macht er sich lustig. Die geistige Überlegenheit, die der Witz plakatiert, ist Seelenbalsam gegen die Erniedrigung" (Joffe 2015: 39). ${ }^{24}$ Selbst wenn der Humor als Heilmittel wirken könnte, ${ }^{25}$ gibt es im Roman Figuren, die ihre traumatische Vergangenheit nicht loswerden können: Der Teilacher Michel Deutscher ,war ein melancholischer Mensch und machte sich bittere Vorwürfe, dass er als Einziger seiner Familie den Horror überlebt hatte“" (Machl: 117-118). Seine Kollegen versuchen ständig, ihm zu helfen und ihn davon zu überzeugen, dass er seinem eigenen Schicksal nicht widersprechen solle und verpflichtet sei, aus seinem Leben etwas zu machen (Machl: 118). Das ist aber nicht allen gelungen, so hatte sich der Teilacher Moische Krautberg 1949 das Leben genommen, weil er die Erinnerung an Treblinka nicht länger aushalten konnte (Machl: 120). Lachen ist somit nicht immer möglich, weil viele nicht über genügend Kraft für die Bewältigung ihres Traumas verfügen.

Während der erste Band der Trilogie die Welt der schelmenhaften Teilacher darstellt, stehen in Machloikes der Humor und seine Transzendenz im Mittelpunkt. Frankfurt, 1953, die schwierigsten Zeiten sind schon vorbei und die Deutschen (die Teilacher eingeschlossen) können schon die ersten Signale des Wirtschaftswunders wahrnehmen. Und nicht zuletzt diese neue verbesserte wirtschaftliche Lage hat dazu beigetragen, dass viele Überlebende und ihre Familien in Deutschland geblieben sind. Obwohl ihre deutschen Nachbarn eine andersartige Perzeption haben von dem, was ab 1933 passiert ist, ${ }^{26}$ versucht jeder in der neuen Republik gut zurecht zu kommen. Die meisten Hauptfiguren haben sich also im Land der ehemaligen Feinde eingerichtet: Einige ziehen weiter von Tür zu Tür, andere haben sogar Geschäfte eröffnet. Aber die Vergangenheit ist immer noch da. In diesem Kontext wird Robert Fränkel, ${ }^{27}$ der einen Tep-

23 Josef Joffe bezieht sich hier auf die Juden als Unterlegene: Das traf zunächst insbesondere auf die Juden des Schtetls in Osteuropa zu, aber später auch auf ihre Glaubensgenossen im Westen, die schon emanzipiert waren (Joffe 2015: 38).

24 In diesem Zusammenhang erklärt der populäre israelische Stand-up-Comedian Gil Kopatch: „Der jüdische Witz war die Waffe der Schwachen: Er war vorsichtig, subtil, andeutend, aber präzise“ (Alexander 2005: 13).

25 In Der Witz und seine Beziehung zum Unbewußten (1905) und in dem 22 Jahre später erschienenen Werk Der Humor (1927) hatte Sigmund Freud auf die Wirkung der Komik auf die menschliche Psyche hingewiesen, und dadurch die Basis für die Entlastungstheorien des Komischen gesetzt: Durch den Witz können psychische Hemmungen abgebaut werden (Freud 1985: 103 ff.).

26 Die Umfragen der Nachkriegszeit zeigen, dass sich die befragten Deutschen nicht betroffen fühlten, was die Schuld am Völkermord anbelangte. Die Verantwortung dafür mussten nur die wichtigsten Figuren des Nationalsozialismus übernehmen. Diesbezüglich ,hielt sich bis in die späten 60er Jahre das zunächst unausrottbare Klischee, daß der Nationalsozialismus eine gute, aber schlecht ausgeführte Idee gewesen sei“ (Wehler 2008: 295).

27 In Die Teilacher erfahren wir, dass er aus einer merkwürdigen Künstlerfamilie stammt: „Mein Vater war Klavierclown, meine Mutter Wahrsagerin, meine Schwester Tänzerin, und alle waren sie Künstler. Hungerkünstler“ (Teilch: 182). Vor dem Krieg hatte Robert Fränkel seinerseits als Schauspieler, Kabarettist und Ansager gearbeitet (Machl: 41). 
pichladen ${ }^{28}$ besitzt, von einem CIA-Beamten vernommen, denn er habe angeblich mit den Nazis kollaboriert: Warum tauche sein Name in so vielen Akten der SS auf? ${ }^{29}$ Diese Verhöre bilden den Kern des Romans, und zugleich werfen Fränkels Aussagen die Frage auf, inwiefern Humor und Völkermord bzw. Totalitarismus kombiniert werden können. Er habe gar nicht mit den Nazis kollaboriert, ihm sei befohlen worden, dem Führer beizubringen, Witze zu erzählen. Darauf wird schon im ersten Teil der Trilogie höhnisch hingewiesen: „Fränkel sollte Hitler ein paar Witze beibringen, damit der in Zukunft auch auf dem Gebiet der fröhlichen Unterhaltung siegen würde! Eine schwierige Aufgabe, denn der Humor des Führers war allgemein bekannt" (Teilch: 183). Ein Beispiel für den rücksichtslosen Sarkasmus, den der Roman manchmal aufweist, stellt die Stelle dar, in der die zwei größten Herzwünsche des Führers erwähnt werden: Die Vernichtung der Juden zu erreichen und einen Witz gut erzählen zu können. Das erfährt Robert Fränkel bei seiner Verabschiedung von dem SS-Mann, der ihm Hitlers Ausbildung anvertraut hat (Machl: 128).

Das ist also Machloikes, ein Roman, in dem mit den Grundlagen des Humors gespielt wird: Schon die Idee, dass Adolf Hitler von einem Juden Witze lernen soll, bedarf unbedingt viel scharfer Ironie und eines beißenden Sarkasmus. Außerdem ist diese Idee selbst schon ziemlich parodistisch und erinnert an andere Werke, in denen die Figur des Führers persifliert wird. ${ }^{30}$ In der Hitler-Komödie Mein Führer - Die wirklich wahrste Wahrheit über Adolf Hitler (2007) soll ein von Goebbels aus dem KZ freigelassener jüdischer Schauspiellehrer (Professor Adolf Grünbaum) die Rhetorik Hitlers schärfen; stattdessen wird er zu einer Art Therapeut, der Hitler im Krisenfall behandelt. In beiden Werken soll ein jüdischer Inhaftierter Kontakt mit dem Führer aufnehmen; im Roman werden jedoch die Pläne schließlich nicht durchgeführt, sodass die Zusammenarbeit zwischen dem jüdischen Lehrer und Hitler selbst im fiktiven literarischen Rahmen nur als absurde Vorstellung existiert. In Machloikes gibt es ebenso wie in Mein Führer eine Inkongruenz, die das Komische bewirkt, nämlich, dass Hitler, dem Inbegriff des Bösen, von einem Juden geholfen werden soll: Im Film geht es um eine psychologische Behandlung; demgegenüber soll Robert Fränkel im Roman Hitler befähigen, mit Anmut Witze zu erzählen. ${ }^{31}$ Die Inkongruenz-

28 Unter den Verkäufern, die Fränkel eingestellt hat, befindet sich Samuel Honigbaum, ein Auschwitz-Überlebender, der im Krankenbau des Lagers Listen für Josef Mengele führen musste (Machl: 33-34). Honigbaum war 1947 im Prozess gegen den Kommandanten des Lagers Rudolf Höß als Zeuge geladen worden. Der Angeklagte griff auf das Argument des Befehlsnotstands als Rechtfertigung für seine Verbrechen zurück (Machl: 35); durch diese Figur wird im Roman das Thema der Rechtsprechung in der Nachkriegszeit bezüglich des Holocaust dargestellt. Für die rechtliche Behandlung des Völkermords war der Gebrauch des Befehlsnotstands als grundlegende Argumentation für die Verteidigung von zentraler Bedeutung (Vgl.: Fischer/ Lorenz 2007: 145-147).

29 „Ihr Name erscheint unter dem Rubrum »Geheime Reichssache« und der Zeit zwischen dem 10. Mai 1944 und 30. August 1944 in zahlreichen Aktennotizen, Schriftstücken und Dokumenten der SS, des Reichssicherheitshauptamts, der Reichskanzlei und in Protokollen des Ministeriums für Volksaufklärung“ (Machl: 57).

30 Schon in den 40er Jahren hatte Charles Chaplin die Figur des Führers in seinem Film The Great Dictator (1940) parodiert, und vor kurzem hat der deutsche Autor Timur Vermes in seinem Roman Er ist wieder da (2012) ein respektloses Bild Hitlers gezeichnet. 2015 wurde diese Geschichte eines auferweckten Führers, der zum Fernsehstar wird, auch verfilmt.

31 Eine andere Gemeinsamkeit der beiden Werke stellt die von den Hauptfiguren getroffene Entscheidung, Hitler zu töten dar (Machl: 154); es handelt sich aber um einen Plan, der in keinem der beiden Fälle durchgeführt wird. Trotzdem träumt Robert Fränkel in Machloikes davon, den Führer tatsächlich ermordet zu haben: „Ich tat mir auch leid, und vor allem tat mir das Schwein leid. Wissen Sie, ich konnte mir ausmalen, wie ich Hitler abgestochen hätte, wie ich genüsslich das Messer in seinem Herzen herumgedreht hätte, wie ich ihm vorher noch die Augen ausgestochen hätte und den Schwanz abgeschnitten, no problem“(Machl: 245). Durch den gescheiterten 
theorien zur Komik erklären, ,dass das Komische aus dem inkongruenten Verhältnis mindestens zweier untereinander inkompatibler Elemente entstehe, von Elementen also, die normalerweise nicht in einer engen Beziehung stehen oder im gleichen Kontext auftreten“ (Sruk 2017: 22). Zum witzigen Ergebnis trägt auch dazu bei, dass die Figur des Juden teilweise eine beherrschende Position einnimmt, denn er ist derjenige, der jemandem etwas lehren kann. Die Unfähigkeit des Führers wird allerdings heruntergespielt, weil ,Witze erzählen ja kein Akt sonderlicher Intelligenz ist" (Machl: 125), das heißt, dass dieser Mangel keinen Makel in seiner Perfektion bedeutet.

Robert Fränkel präsentiert sich als ein Profi des Witzes: „Ich war ein professioneller Witzeerzähler, manchmal auch ein Witzeerfinder. Die Leute riefen mir Begriffe zu, aus dem Publikum, und ich machte irgendwelche lustigen Geschichten daraus“ (Machl: 61). Während der von den Amerikanern durchgeführten Vernehmung macht Fränkel deutlich, dass er den Nationalsozialismus schon vor dem Krieg ausgelacht hatte: Für ihn stelle der Hitlergruß keine Schwierigkeit dar, da er ihn im Kabarett geübt habe (Machl: 123). Jahre später, im KZ, hat ihm seine Fähigkeit letzten Endes das Leben gerettet, und darin liegt auch die Besonderheit der Handlung: Der Humor und seine Verwirklichung - der Witz - haben über zwei unterschiedliche Wege seinen Sturz in den Abgrund vermieden, indem sie seine psychische ebenso wie seine körperliche Existenz gesund gehalten haben. Mehr kann man von einem Witz nicht verlangen.

\section{Das letzte Paradox: Versöhnung damals und heute}

Die Trilogie zeigt, dass es auch Raum für eine Versöhnung gibt. Schon damals, in den ersten Jahren nach dem Krieg, ist sie möglich, wie diverse Liebesgeschichten zwischen Juden und Nichtjuden bezeugen. ${ }^{32}$ Laut Garrick führt der Humor oft zur Vergebung (Garrick 2006: 173), und diese dürfte einen Schritt weiter zu einem friedlichen Treffen mit den ehemaligen Feinden darstellen. Diesbezüglich wird in der Trilogie implizit eine Verbindung zwischen einem möglichen Aussöhnungsprozess und dem Humor hergestellt, denn dieser begünstigt gewissermaßen warmherzige Beziehungen zwischen Juden und Nichtjuden; auf jeden Fall scheint es, dass beinahe nur die Liebe bestimmte Hindernisse überwinden kann. Else Adam, die ein kleines Kohlen-Geschäft betreibt, erzählt dem Juden Max Holzmann von ihrer Kindheit,

\footnotetext{
Mord hätte er die Frustration, die Verzweiflung und den ganzen Groll seiner Glaubensbrüder loswerden können. Wie die Trilogie zeigt, bleibt ihnen ein schwacher Trost: Der Führer ist tot, und sie leben immer noch, als Erinnerung an dessen Niederlage.

32 Jenseits der Liebe gibt es auch Figuren im Roman, die diese Versöhnung betreiben, um mit ihrer eigenen familiären Vergangenheit zurecht zu kommen. Jürgen Kelbassa, einer der für Robert Fränkel arbeitenden Verkäufer, stammt ,aus einer unbelehrbaren Nazifamilie“ und diese Belastung will er dadurch überwinden, dass er eine „Liebe zu Juden schon gnadenlos und pathologisch“ hat (Machl: 36). Mittels dieser Figur wird in der Trilogie ein üblicher Menschentyp der Epoche dargestellt, wobei eine charakteristische Entwicklung vom Nationalsozialismus zum Kommunismus aufgezeigt wird: „Kelbassa, einst als Hitlerjunge gestartet, als Volkssturmpimpf den Krieg gemeistert, dann als Falke den sozialistischen Werktätigen zugeflogen, war ein typisches Kind seiner Zeit, hin- und hergerissen in seinen politischen Ansichten“ (Machl: 37). Aus seiner Position gegenüber dem Kommunismus ergeben sich Gewissenskonflikte, zum Beispiel in Bezug auf den Aufstand am 17. Juni 1953 in Berlin, da Kelbassa „,noch nicht recht wusste, ob er sich mit den aufständischen Arbeitern solidarisieren oder die stalinistischen Genossen unterstützen sollte“ (Machl: 44).
} 
von ihrem im Krieg verschwundenen Verlobten und von den furchtbaren Bombenangriffen in der Stadt:

Max hatte sich diese Geschichten bisher mitleidlos angehört, denn das Schicksal der Deutschen berührte ihn kaum. Nicht, dass er kein Gefühl für das Leiden anderer empfunden hätte, im Gegenteil, er war sensibel, aber bei den Deutschen versteinerte sich sein Herz. Doch wie er Else nun so gegenübersaß und in ihre lebhaften, ehrlichen Augen schaute, wie er ihr zuhörte, wie sie mit ihrer sanften Stimme sprach, da kam ihm der Gedanke, wie schade es doch gewesen wäre, wenn eine Bombe die schöne Else zerrissen oder ein einstürzendes Haus sie begraben hätte (Teilch: 199).

Holzmann war ein polnischer Jude, der seine ganze Familie in Auschwitz verloren hatte und dessen Ziel Amerika war. Sein zunächst vorläufiger Aufenthalt in Deutschland wird definitiv, denn unerwartet ,lernte er ausgerechnet eine junge deutsche Frau kennen, Else Adam, und sie verliebten sich ineinander und haben schließlich geheiratet, nachdem Else konvertiert war" (Machl: 186).

Dagegen veranschaulicht ein anderes Ehepaar, die Unterleitners, die Figur des ,guten Deutschen", des Judenretters. Sie hatten sich in Paris verliebt, als er Oberleutnant war und sie sich im Exil befand. Es ist dem Offizier gelungen, ihr das Leben zu retten, und nach dem Krieg sind sie nach Frankfurt gezogen, um ein Café zu führen, wo die Teilacher stundenlang sitzen (Teilch: 94). Zusätzlich zur Liebe ist also auch der Einsatz einiger deutscher Bürger für die Verfolgten eine beachtliche Grundlage für eine hypothetische Versöhnung zwischen Juden und Nichtjuden. ${ }^{33}$ Für einige jüdische Überlebende wurde die Dankbarkeit gegenüber denjenigen, die ihnen während des Holocaust geholfen hatten, zum Hauptargument ihrer Entscheidung, in Deutschland zu bleiben. Das ist zwar nicht der zentrale Punkt der Trilogie Bergmanns, aber das wurde nach 1990 in zahlreichen deutschsprachigen Werken dargestellt; Ursache dafür war nicht zuletzt die Auswirkung des amerikanischen Films über Oskar Schindler vom Jahr 1993 auf das kollektive Gedächtnis. ${ }^{34}$ Diesbezüglich erläutert Peter Schneider: „Bis zu Spielbergs Film Schindlers Liste sind die Geschichten der Helfer - allen Anstrengungen der Geretteten zum Trotz - nicht ins Bewusstsein einer breiteren deutschen Öffentlichkeit vorgedrungen" (Schneider 2001: 12). Insbesondere ab diesem Zeitpunkt werden Begriffe wie „der gute Deutsche“ oder „,der andere Deutsche“ populär, die diese Personen bezeichnen, die einen aktiven Widerstand gegen die Verfolgung der Juden leisteten und deren Verhalten damit vom allgemeinen Antisemitismus abwich. Der deutsche Autor Peter Schneider schreibt einen Roman über den deutsch-jüdischen Musiker Konrad Latte: "Und wenn wir nur eine Stunde gewinnen». Wie ein jüdischer Musiker die Nazi-Jahre überlebte (2001). Latte konnte dank der Hilfe unzähliger Helfer den Holocaust im Untergrund überleben, was dazu beitrug, dass er nach 1945 das Land seiner Verfolger (aber auch seiner Wohltäter) nicht verließ: "Was Konrad und Ellen davon abgehalten hat, aus Deutschland auszuwandern, war die Verbundenheit mit jenen etwa fünfzig , anderen“ Deutschen, die ihm über zwei Jahre lang Tag für Tag geholfen haben, den Nazi-Mördern zu entgehen“(Schneider 2001: 151). In Zusammenhang mit diesen, ,anderen Deut-

33 Ein ähnlicher Vorfall wird im oben erwähnten Werk Familienleben präsentiert, der die Liebesgeschichte zwischen einer Holocaustüberlebenden und deren Retter darstellt. In diesem Fall hat die Fiktion einen autobiographischen Hintergrund, da die Autorin Viola Roggenkamp die Tochter des den Roman inspirierenden Ehepaares ist.

34 Siehe: Martín Martín 2013. 
schen" wird im zweiten Band der Trilogie der Arzt Georg Fuchs präsentiert, der seine Dissertation zurückzog, als sein jüdischer Doktorvater 1933 vom Dienst suspendiert worden war und sich danach weigerte in den NSDÄB, den Nationalistischen Deutschen Ärztebund, einzutreten, was zur Folge hatte, dass er in jenen Jahren nicht als Arzt arbeiten durfte (Machl: 75). 1943 desertierte er sogar und erst 1945 kam er mit der US-Armee nach Deutschland zurück, um endlich wieder als Arzt zu arbeiten (Mach: 76). Diese Figuren ermöglichen ein ermutigenderes Panorama in der deutschen Gesellschaft, in der die Teilacher gut zurecht zu kommen lernen müssen.

Jahrzehnte später, schon im 21. Jahrhundert, als die Generation der Teilacher schon alt geworden ist, erzählt der dritte Band der Trilogie (Herr Klee und Herr Feld) von den letzten Jahren der zwei Figuren, die dem ganzen Werk eine einheitliche Gestalt verleihen: Moritz und Alfred Kleefeld (Freddy Clay). Sie sind der rote Faden, der die drei Teile der fiktionalen Welt ebenso wie die jeweiligen chronologischen Epochen miteinander verbindet: In Die Teilacher als Kinder, die ins Exil nach Amerika gehen müssen; in Machloikes als Jugendliche, die in einem blühenden Deutschland aufwachsen; schließlich in Herr Klee und Herr Feld als Ältere, die sich gegenseitig Gesellschaft leisten, nachdem ihre Leben völlig unterschiedlich verlaufen sind. Die Brüder kommen aus Amerika (Moritz) bzw. aus Italien (Alfred) nach Frankfurt zurück, um dort ihre letzten Jahre zu verbringen. Beide hatten jahrelang im Ausland Karriere gemacht: Moritz als Professor und Alfred als Darsteller in B-Movies. Aber letztendlich bleibt Deutschland ihre Heimat, selbst wenn Alfred vor fünfzig Jahren anderer Meinung war: „Die Juden lebten zwar unter den Deutschen, mit den Deutschen lebten sie nicht. Die Mentalitäten waren zu verschieden, es gab keine gemeinsame Erinnerung. Und vor allem, es gab kein Verzeihen“ (Machl. 316). Im dritten Teil der Trilogie zeigt sich deutlich, dass man eine Einigung mit den „Feinden" erzielen kann, da sich die Brüder Kleefeld in ihr palästinensisches Dienstmädchen gewissermaßen verlieben, und dadurch einige ihrer Anschauungen in Frage stellen müssen. Die jeweiligen Vorurteile werden größtenteils abgebaut, indem alle drei versuchen, die Gegenargumente nachzuvollziehen. Sie werden zumindest dazu veranlasst, an Diskussionen teilzunehmen, bei denen gegenteilige Ansichten geäußert werden. Folgender Dialog ist ein gutes Beispiel dafür:

Zamira schaute ihn ernst an und sagte:

Heute behandeln die Juden die Palästinenser so, wie die Nazis die Juden. Die Juden sollten doch wissen, wie das ist.

Erstens ist das nicht wahr. Gaza oder das Westjordanland mit einem KZ zu vergleichen, ist infam, schoss Moritz zurück. Zweitens: Auschwitz war keine Universität, dort hat man nicht Humanität gelehrt. Warum erwartet man immer von den Juden Verständnis und Nachgiebigkeit? Sie sind ein Volk wie jedes andere. So gut und so schlecht.

Die Stimmung hatte sich verändert. Beide spürten das. Zamira schaute auf die Uhr. Hoffentlich behält sie das Marmeladenglas, dachte Moritz.

Sie stellte es auf die Konsole.

Ich muss überlegen, sagte sie, ich melde mich.

Nachdem sie gegangen war, musste Moritz lange an sie denken. Nein, sie war keine eingeschleuste Terroristin. Er hoffte, sie würde den Job annehmen (KuF: 79).

Zamira wird später mit dem streng kontrollierten Antisemitismus in Deutschland konfrontiert, als sie eine von der Polizei strikt beaufsichtigte jüdische Schule beob- 
achtet (KuF: 138-139); Moritz und Alfred ihrerseits hören aufmerksam zu, wenn Zamira von ihren Erfahrungen in Palästina und dem Libanon berichtet, was für die Brüder Kleefeld eine neuartige und beunruhigende Perspektive bedeutet. Hier entsteht teilweise ein Parallelismus zu der früheren Beziehung zwischen Deutschen und Juden, die unversöhnlich aussah. Die Figur Zamiras ermöglicht, den israelisch-palästinensischen Konflikt und demnach das problematische Verhältnis zwischen Juden und Muslimen, sowie ihren Einfluss auf die öffentliche Meinung zu veranschaulichen.

\section{Fazit}

Der Autor Michel Bergmann ist Sohn von deutsch-jüdischen Überlebenden des Holocaust, was in seiner Teilacher-Trilogie von entscheidender Bedeutung ist. Wie in diesem Beitrag dargelegt, behandelt das Werk einige für deutschsprachige jüdische Autoren der zweiten Generation typische Themen, darunter das deutsch-jüdische Verhältnis oder die Frage nach der eigenen Identität. Andererseits kann die Trilogie der nach 1990 florierenden Gattung der Familien- oder Generationenromane zugeordnet werden. Nach Aleida Assmann ,steht der Familienroman im Zeichen der Kontinuität“ (Assmann 2006a: 26), es handelt sich nämlich dabei um ein Genre, das es den Autoren ermöglicht, einen persönlichen Platz in der eigenen familiären Geschichte zu finden. Und das ist sicherlich eines der Ziele von Bergmanns Gesamtwerk.

Neben einer literaturwissenschaftlichen Analyse beschreibt dieser Artikel die historischen und kulturellen Zusammenhänge, durch die diese von biographischen Referenzen durchzogenen Romane angemessen nachvollzogen werden können. Die Teilacher hatten keinerlei Hemmungen, ihre Mitbürger zu betrügen, denn letzten Endes gehörten diese ja dem Volk der Täter an. Die historischen Quellen bestätigen, dass die meisten jüdischen Überlebenden nach 1945 Deutschland nur als eine vorläufige Station sahen, sodass moralische Bedenken der Teilacher gegenüber den deutschen Nachbarn keine große Rolle spielten. Das Zurückgreifen Bergmanns auf den Humor als zentrales Element der Trilogie ist kein rein stilistisches Mittel, denn Ironie, Lachen und Sarkasmus dienten den in Deutschland gebliebenen Juden sowohl zur Traumabewältigung als auch zum Schutz gegenüber einer oft feindseligen Gesellschaft. Die Bedeutung des Humors wurde im Artikel u. a. am Beispiel klassischer Schriften (Bergson, Freud) und des Berichts Viktor Frankls (Psychiater und KZ-Überlebender) dargestellt. In den letzten Jahrzehnten belegen mehrere Filme und literarische Werke diese Verbindung vom Humor und Holocaust, was eine neue Phase im Umgang mit dem Völkermord an den Juden einläutet.

Ein weiterer relevanter Aspekt der Trilogie ist die Thematisierung der Gründe, die einige jüdische Überlebende zur Versöhnung mit dem Volk der ehemaligen Täter bewegen. Man kann hier zwei Hauptbeweggründe unterscheiden: Zum einen die Liebe und zum anderen die Dankbarkeit gegenüber denjenigen Deutschen, die sie vor dem Tod gerettet hatten. Der zweite Themenbereich hatte nach dem Erfolg des Filmes Schindler's List (1993) an Bedeutung gewonnen, was den Weg für zahlreiche fiktionale - und nicht-fiktionale - damit zusammenhängende Werke ebnete. Dieser Artikel ist im Rahmen der kulturellen Studien anzusiedeln und macht deutlich, dass sich die Teilacher-Trilogie sowohl auf die persönliche bzw. familiäre Erfahrung des Autors als auch auf die den aktuellen historisch-kulturellen Kontext kennzeichnenden Verhältnisse stützt. 


\section{Literaturverzeichnis}

Adenauer, K., «Erste Regierungserklärung» 1949, https://www.konrad-adenauer.de/dokumente/erklaerungen/1949-09-20-regierungserklaerung [25.04.2018].

Adorno, T., Kulturkritik und Gesellschaft I. Frankfurt am Main: suhrkamp taschenbuch wissenschaft 2003 [1951].

Alexander, R., «Auschwitz ist ein Witz», taz 3.5.2005, 13.

Arndt, S. (Produzent) / Levy, D. (Regisseur), Mein Führer - Die wirklich wahrste Wahrheit über Adolf Hitler. Deutschland: Stefan Arndt, X Filme 2007.

Assmann, A., Generationsidentitäten und Vorurteilsstrukturen in der neuen deutschen Erinnerungsliteratur. Wien: Picus Verlag 2006a.

Assmann, A., Der lange Schatten der Vergangenheit. Erinnerungskultur und Geschichtspolitik. München: C. H. Beck 2006b.

Bergmann, M., Die Teilacher. München: dtv 2011 [2010].

Bergmann, M., Machloikes. München: dtv 2013 [2011].

Bergmann, M., Herr Klee und Herr Feld. München: dtv 2014 [2013].

Bergson, H., Das Lachen. Ein Essay über die Bedeutung des Komischen. Hamburg: Felix Meiner Verlag 2011 [1900].

Brenner, M., Nach dem Holocaust. Juden in Deutschland 1945-1950. München: C. H. Beck 1995.

Chaplin, C. (Produzent und Regisseur), The Great Dictator. USA: Charles Chaplin Film Corporation 1940.

Dückers, T., Himmelskörper. Berlin: Aufbau Verlag 2003.

Fischer, T. / Lorenz, M. (Hg.), Lexikon der »Vergangenheitsbewältigung « in Deutschland. Debatten- und Diskursgeschichte des Nationalsozialismus nach 1945. Bielefeld: transcript Verlag 2007.

Forte, D., Tetralogie der Erinnerung (Das Muster, Der Junge mit den blutigen Schuhen [Tagundnachtgleiche], In der Erinnerung, Auf der anderen Seite der Welt). Frankfurt am Main: Fischer 1992, 1995, 1998, 2004.

Frankl, V. E., ...trotzdem Ja zum Leben sagen. Ein Psychologe erlebt das Konzentrationslager. München: Deutscher Taschenbuch Verlag 1982 [1977].

Freud, S., Der Witz und seine Beziehung zum Unbewußten. Frankfurt am Main: Fischer Verlag 1985 [1905].

Garrick, J., «The Humor of Trauma Survivors: Its Application in a Therapeutic Milieu», Journal of Aggression, Maltreatment \& Trauma Vol. 12 (2006), 169-182.

Grossmann, A., Jews, Germans, and Allies. Close Encounters in Occupied Germany. Princeton: Princeton University Press 2007.

Herzberger, J., Erinnerungsarbeit der Holocaustliteratur der zweiten Generation. Göttingen: Optimus Mustafa Verlag 2009.

Jahn, B., «Familienkonstruktionen 2005. Zum Problem des Zusammenhangs der Generationen im aktuellen Familienroman», Zeitschrift für Germanistik, XVI - 3/2006, 581-596.

Joffe, J., Mach dich nicht so klein, du bist nicht so gross! Der jüdische Humor als Weisheit, Witz und Waffe. München: Siedler 2015.

Martín Martín, J. M., «La banalidad del bien: sobre stille Helden y literatura», RdFA (Revista de Filología Alemana) Vol. 21 (2013), 85-102.

Menasse, E., Vienna. Köln: Kiepenheuer und Witsch 2005.

Roggenkamp, V., Familienleben. Frankfurt am Main: Fischer 2004. 
Schneider, P., «Und wenn wir nur eine Stunde gewinnen». Wie ein jüdischer Musiker die Nazi-Jahre überlebte. Berlin: Rowohlt 2001.

Schneider, R. C., Wir sind da! Die Geschichte der Juden in Deutschland von 1945 bis heute. Berlin: Ullstein 2000.

Sruk, M., Lachen nach Auschwitz? Herausforderungen der Filmkomödie zum Holocaust (Doktorarbeit). Gießen: GEB (2017), http://geb.uni-giessen.de/geb/volltexte/2017/12608/ [15.03.2018].

Steinecke, H., Literatur als Gedächtnis der Shoah. Deutschsprachige jüdische Schriftstellerinnen und Schriftsteller der ,zweiten Generation “. Paderborn: Verlag Ferdinand Schöningh 2005.

Vermes, T., Er ist wieder da. Köln: Eichborn Verlag 2012.

Wassermann, J., Mein Weg als Deutscher und Jude. München: dtv 1994 [1921].

Wehler, H., Deutscher Gesellschaftsgeschichte. Bundesrepublik und DDR 1949-1990. München: C. H. Beck 2008. 